\title{
Thermal stability and partial dewetting of crystalline organic thin films: 3,4,9,10-perylenetetracarboxylic dianhydride on $\mathrm{Ag}(111)$
}

\author{
B. Krause, ${ }^{\text {a) }}$ A. C. Dürr, F. Schreiber, ${ }^{\text {b) }}$ and H. Dosch ${ }^{\text {c) }}$ \\ Max-Planck-Institut für Metallforschung, Heisenbergstr. 3, D-70569 Stuttgart, Germany \\ O. H. Seeck \\ IFF, Forschungszentrum Jülich GmbH, D-52425 Jülich, Germany
}

(Received 7 March 2003; accepted 13 May 2003)

\begin{abstract}
The thermal stability and dewetting effects of crystalline organic thin films on inorganic substrates have been investigated for a model system for organic epitaxy, 3,4,9,10-perylenetetracarboxylic dianhydride (PTCDA) on $\mathrm{Ag}(111)$. The thin films deposited under a variety of growth conditions have been annealed stepwise and studied by in situ x-ray diffraction and noncontact atomic force microscopy. It has been found that comparatively smooth films deposited at temperatures $T_{g}$ $\$ 350 \mathrm{~K}$ are metastable, while films deposited at $T_{g} \gtrsim 350 \mathrm{~K}$ are stable against annealing on a time scale of several hours. The thermal expansion of thin PTCDA films and bulk samples has been determined. (C) 2003 American Institute of Physics. [DOI: 10.1063/1.1589471]
\end{abstract}

\section{INTRODUCTION}

Due to their attractive electronic and optoelectronic properties, crystalline organic semiconductors, such as oligoacenes, perylenes, and phthalocyanines, are presently receiving much attention. Since their performance depends strongly on the structural definition, much effort has been devoted to controlling the structural quality of these systems. ${ }^{1,2}$ In view of possible applications, however, not only the room-temperature structure, but also phenomena related to the thermal behavior, such as thermal stability, possible dewetting effects of thin films, and interdiffusion at interfaces, need to be investigated (see Ref. 3 and references therein) which are generally not well understood for organic molecular crystals and thin films.

Besides its relevance for the functioning and long-time stability of devices, the thermal behavior offers a challenge for the fundamental understanding of these materials. The dewetting effects are strongly related to the crystalline structure of the material and are thus quite different from the wetting-dewetting problems of an isotropic liquid. ${ }^{4}$ Moreover, the thermal expansion is expected to be highly anisotropic. ${ }^{5}$ The situation is complicated due to the fact that many organic molecular crystals may exhibit several distinct crystal structures (polymorphism), which are energetically very similar and may thus coexist. On the other hand, if the structure formation and dewetting mechanism are understood, they may be exploited for the fabrication of nanostructures, such as quantum wells or quantum dots, again, similar to the case of inorganic semiconductors. ${ }^{6}$

In this article, the thermal stability and dewetting effects

\footnotetext{
${ }^{a)}$ Electronic mail: krause@esrf.fr

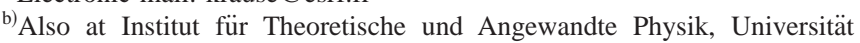
Stuttgart; new address: Physical and Theoretical Chemistry Laboratory, Oxford University, South Parks Road, Oxford, OX1 3QZ, United Kingdom.

${ }^{c}$ Also at Institut für Theoretische und Angewandte Physik, Universität Stuttgart, Pfaffenwaldring 57, D-70550 Stuttgart, Germany.
}

are studied using films of 3,4,9,10-perylenetetracarboxylic dianhydride [(PTCDA) see Fig. 1(a)] grown on $\mathrm{Ag}(111)$ single crystals. PTCDA is considered a model system for crystalline organic semiconductors. ${ }^{1,7-12}$ In the bulk, PTCDA crystallizes in molecular sheets stacked in the PTCDA(102) direction perpendicular to the molecular plane. The unit cell contains two coplanar molecules in a herringbone structure. The two monoclinic bulk polymorphs, $\alpha(a=3.74 \AA$, $b$ $=11.96 \AA, \quad c=17.34 \AA$, and $\left.\beta=98.8^{\circ}\right)^{13,14}$ and $\beta(a$ $=3.87 \AA, b=19.30 \AA, c=10.77 \AA$, and $\left.\beta=83.6^{\circ}\right),{ }^{15,16}$ differ slightly in the stacking of the molecular sheets. The welldefined sheetlike molecular stacking with the molecular planes parallel to the substrate surface and the existence of both polymorphs growing in the PTCDA(102) direction have also been found in thin films of PTCDA/Ag(111), the asgrown structure of which has been studied in detail. ${ }^{7,11,12}$

\section{EXPERIMENT}

The PTCDA films studied here have been deposited by organic molecular-beam epitaxy (MBE) on $\mathrm{Ag}(111) .{ }^{1}$ Prior to deposition, the $\mathrm{Ag}$ substrate has been cleaned by repeated cycles of sputtering (30 min at room temperature, $\mathrm{Ar}^{+}$energy $500-600 \mathrm{eV}$, Ar pressure $\approx 6 \times 10^{-5} \mathrm{mbar}$ ) and annealing at $\approx 700-900 \mathrm{~K}$ for at least $15 \mathrm{~min}^{12}$ The purified and thoroughly outgassed PTCDA has been evaporated from a Knudsen cell kept at approximately $575 \mathrm{~K}$ at deposition rates, $F$, between 0.8 and $6.3 \AA / \mathrm{min}$. The growth temperature, $T_{g}$, has been varied between 135 and $473 \mathrm{~K}$, the film thickness between 30 and $180 \AA$.

After the deposition, temperature-induced changes have been studied by noncontact atomic force microscopy (AFM) and by x-ray diffraction. The AFM experiments have been performed under UHV conditions. For the x-ray experiments, a portable MBE chamber equipped for in situ x-ray diffraction has been used. ${ }^{17}$ The scattering experiments have been performed at the Hamburger Synchrotronstrahlungslabor (HASYLAB) at the beamline W1 with the wavelength 
(a)

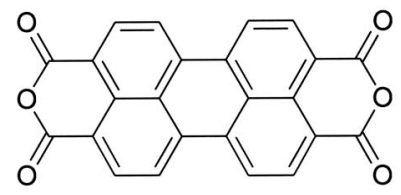

(b)

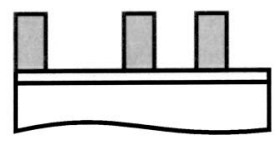

Film

Wetting layer

Substrate

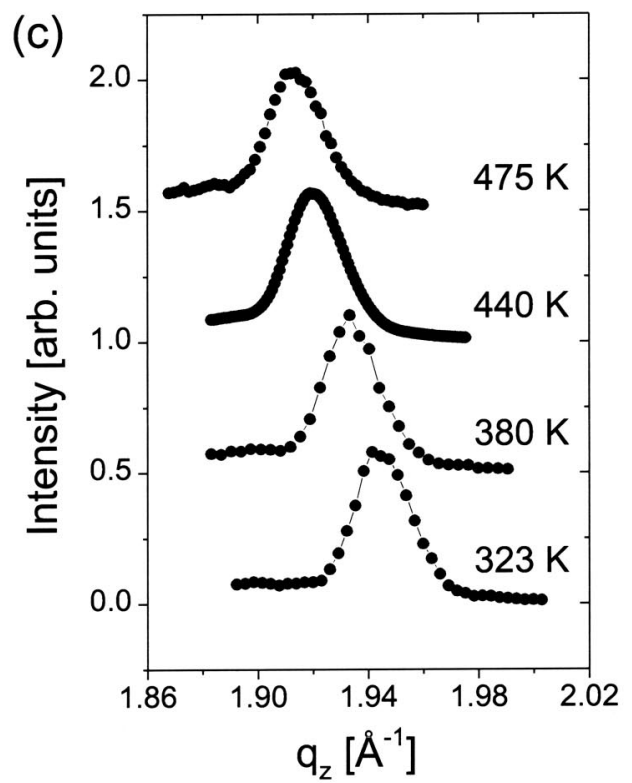

FIG. 1. (a) PTCDA. (b) Schematic of the morphology of a high-temperature film. (c) Intensity of the PTCDA(102) peak as a function of the annealing temperature, $T_{a}$, measured for a sample with $d=256 \AA, T_{g}=384 \mathrm{~K}$, and $F=6.3 \AA / \mathrm{min}$.

$\lambda=1.237 \AA$. Supplementary measurements have been performed at a rotating anode with molybdenum $K \alpha$ radiation. In addition, powder diffraction measurements of the thermal expansion of the PTCDA $\alpha$-phase have been recorded on a film. For this, copper $K \alpha$ radiation has been used.

\section{RESULTS}

The morphology of thin PTCDA films depends strongly on the growth temperature, $T_{g}{ }^{12}$ For $T_{g} \$ 350 \mathrm{~K}$ (lowtemperature regime), the films are relatively smooth, i.e., the local film thickness is similar to the average film thickness, $d$. For $T_{g} \geq 350 \mathrm{~K}$ (high-temperature regime), separate islands grow on a wetting layer [see Fig. 1(b)]. In the following, the results of the study of the thermal stability of thin PTCDA films grown at different $T_{g}$ are presented.

\section{A. Annealing of film growth at high temperature}

Several samples grown in the island regime (large separate islands on a 2 ML thick wetting layer) with the parameters $F=6.3 \AA, d=30-180 \AA$, and $T_{g}=381-473 \mathrm{~K}$ have been annealed in several steps with the step size varying

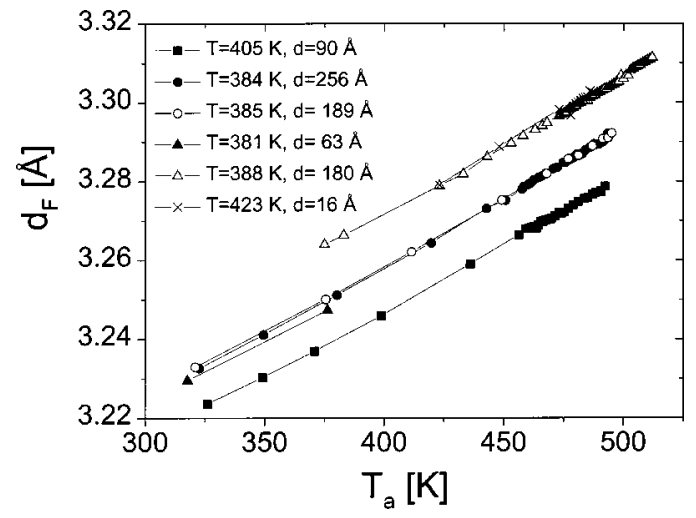

FIG. 2. Thermal expansion, measured for several samples along the PTCDA(102) direction. The offset of the different measurements is due to systematic errors.

between 1 to $40 \mathrm{~K}$. Typically, the samples have been kept at each temperature for $30 \mathrm{~min}$. The annealing process has been studied by x-ray measurements of the specular rod, i.e., with the momentum transfer $\mathbf{q}=q_{z} \mathbf{e}_{z}$ perpendicular to the sample surface. Figure 1(c) shows examples of the PTCDA(102) peak for a sample with $d=256 \AA, T_{g}=384 \mathrm{~K}$, and $F$ $=6.3 \AA / \mathrm{min}$. The sample has been cooled to $323 \mathrm{~K}$, and then annealed up to the desorption of the film. The annealing induces no qualitative change of the Bragg peak. Since the peak width is inversely proportional to the island height [see Fig. 1(b)], this indicates that the morphology of the film is stable during the annealing process. With increasing annealing temperature $\left(T_{a}\right)$ the position of the Bragg peak, $q_{p}$, moves to smaller $q_{z}$ since the out-of-plane lattice parameter $\left(d_{F}\right)$ of the film increases due to thermal expansion.

Figure 2 shows the lattice parameter, $d_{F}=2 \pi / q_{P}$, as a function of the annealing temperature for various samples. The peak position, $q_{P}$, has been determined by Gaussian fits of the Bragg peak after background subtraction. Within the experimental uncertainty, the lattice parameter varies linearly with $T_{a}$ over the temperature range studied. The linear thermal expansion coefficient perpendicular to the sample surface, ${ }^{18}$

$$
\alpha_{\perp}=\frac{1}{d_{F}(298 \mathrm{~K})} \frac{\partial d_{F}}{\partial T},
$$

averaged over several samples grown in the high-temperature (island) regime, is $\alpha_{\perp}=1.05 \pm 0.06 \times 10^{-4} \mathrm{~K}^{-1}$. For the in-plane linear thermal expansion coefficient $\alpha_{\|}$, we can only make an estimate. In Ref. 12, we have shown that the in-plane unit-cell dimensions measured at high $T_{g}$ are $b_{1}$ $=20.11 \pm 0.05 \AA$ and $b_{2}=12.16 \pm 0.05 \AA$ for the PTCDA $\alpha$ phase and $b_{1}=19.44 \pm 0.05 \AA$ and $b_{2}=12.53 \pm 0.05 \AA$ for the PTCDA $\beta$ phase. The angle between $b_{1}$ and $b_{2}$ is $90.0 \pm 0.2^{\circ}$ (rectangular unit mesh). From the comparison of the unit-cell dimensions measured at high $T_{g}$ (Ref. 12) with the rectangular room-temperature structure of the similar bulk phases, we derive the effective linear thermal expansion $\alpha_{\|}=5 \pm 2$ $\times 10^{-5} \mathrm{~K}^{-1}$. Despite the large error bars, this shows an anisotropy of the thermal expansion parallel and perpendicular to the sample surface (see Sec. IV). 
(a) As-grown (at $135 \mathrm{~K}$ )

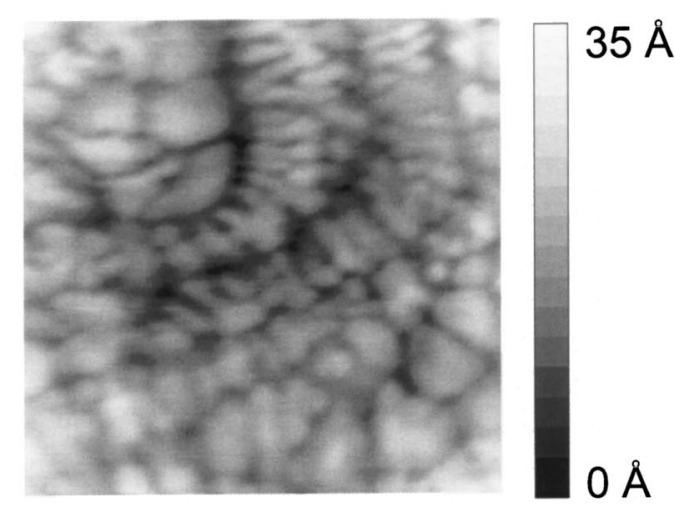

(b) $343 \mathrm{~K}$

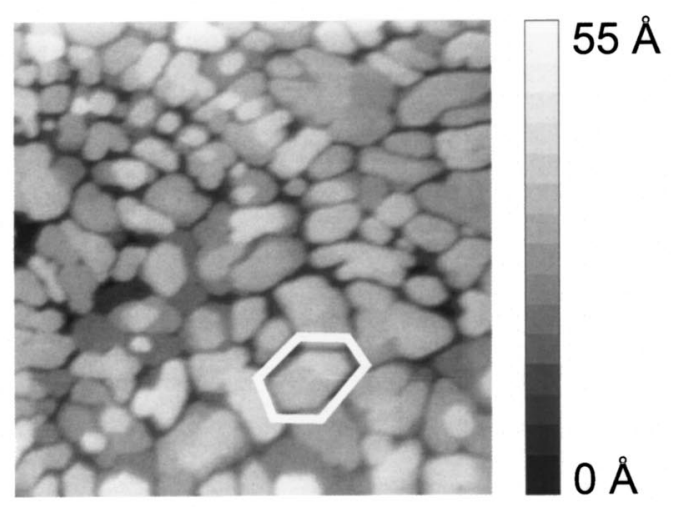

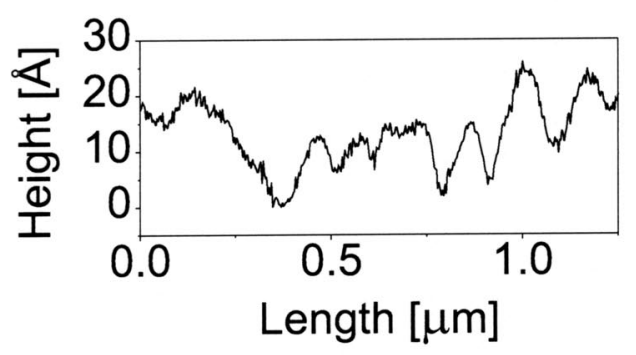

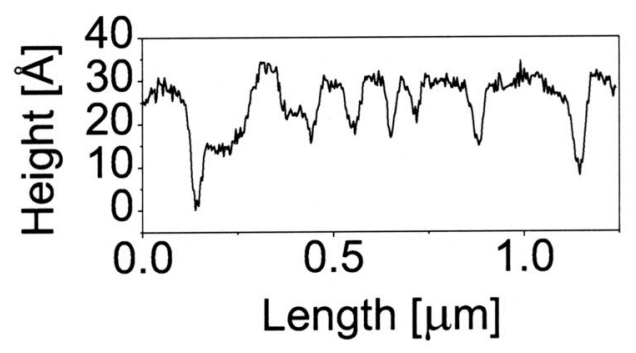

(c) $373 \mathrm{~K}$
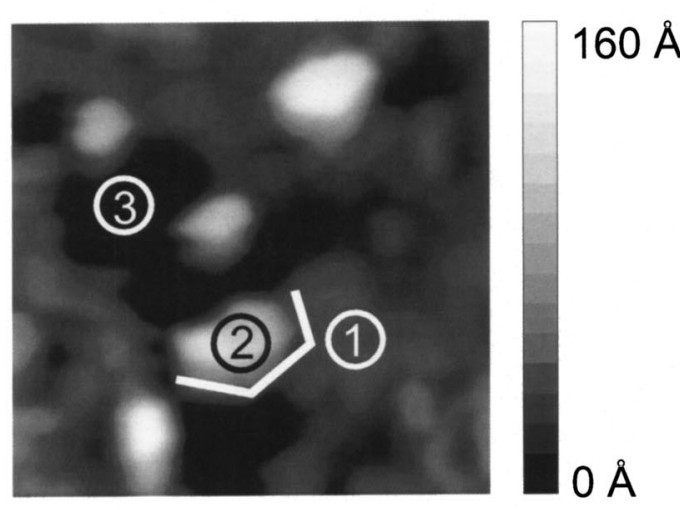

(d) $433 \mathrm{~K}$

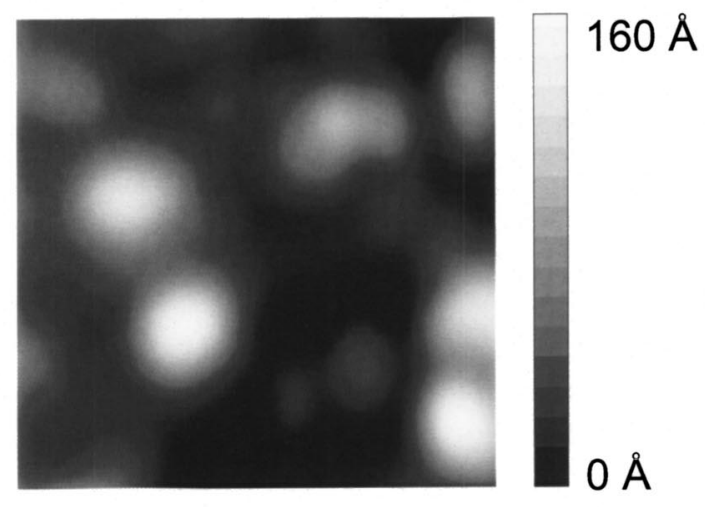

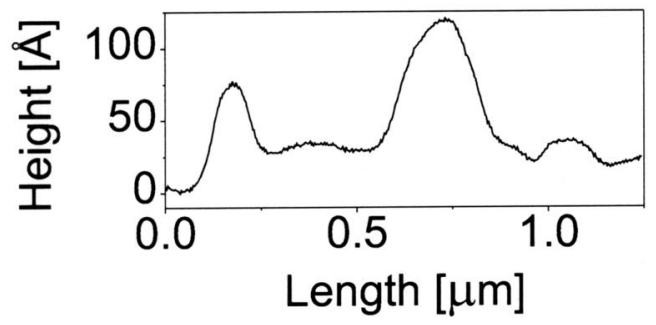

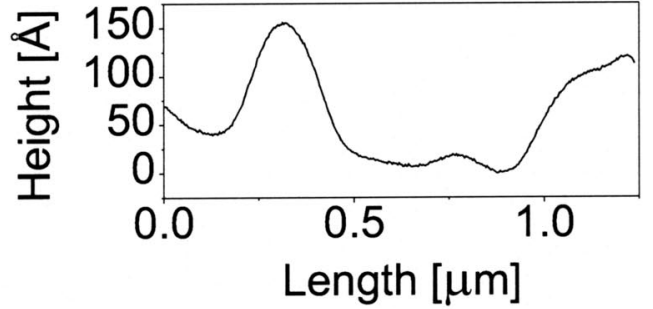

FIG. 3. Annealing of a $50 \AA$ PTCDA film (area of the AFM images $1.25 \times 1.25 \mu \mathrm{m}^{2}$ ) as a function of the annealing temperature. Facets and different morphology regions are indicated.

\section{B. Annealing of films grown at low temperature}

Several PTCDA films have been grown at $T_{g}<350 \mathrm{~K}$, i.e., with a comparatively smooth morphology. The annealing of the samples has been studied by noncontact AFM measurements and by x-ray measurements of the specular rod. While the AFM images give direct information about the local morphology of the films, the x-ray measurements combine morphological and structural information, averaged over the sample surface. 


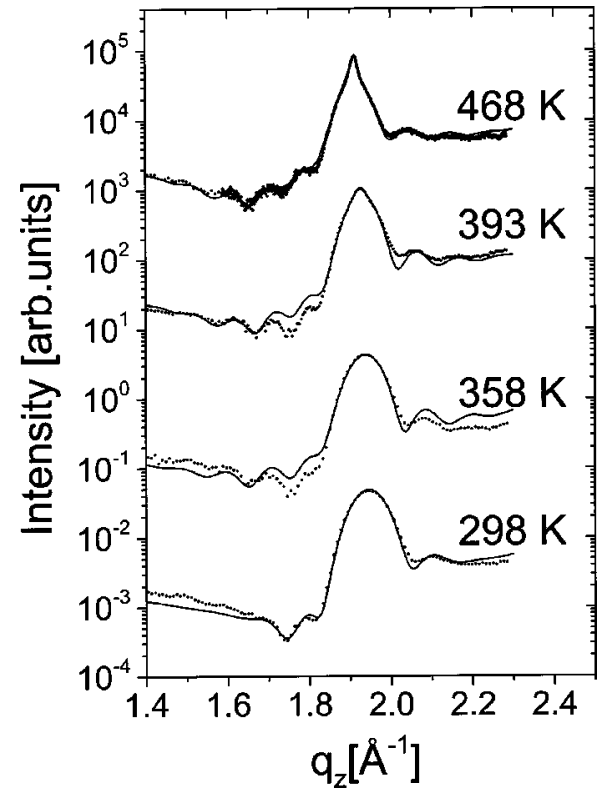

FIG. 4. Measured (circles) and calculated (lines) specular rod intensity as a function of the annealing temperature.

\section{Atomic force microscopy studies}

In order to study the annealing behavior of a relatively smooth PTCDA film by AFM, a sample was deposited at $T_{g}=135 \mathrm{~K}$, with a deposition rate $F=0.8 \AA / \mathrm{min}$ and an average film thickness $d=50 \AA$. The annealing was performed in several $30-40 \mathrm{~K}$ steps up to the desorption of the film (at approximately $500 \mathrm{~K}$ ). The sample was kept for typically $2 \mathrm{~h}$ at each annealing temperature, $T_{a}$. The subsequent AFM measurements were performed at room temperature.

Figure 3 shows examples for the morphology as a function of the annealing temperature. The images have a size of $1.25 \times 1.25 \mu \mathrm{m}^{2}$ and are typical for the morphologies at the respective temperature.

The as-grown morphology [Fig. 3(a)] is characterized by islands with a lateral size of 50-200 nm (see Ref. 12). The island shape is not well defined, and neighboring islands are so close to each other that the surface looks wavy. The roughness of the film is of the order of $2-3$ monolayers

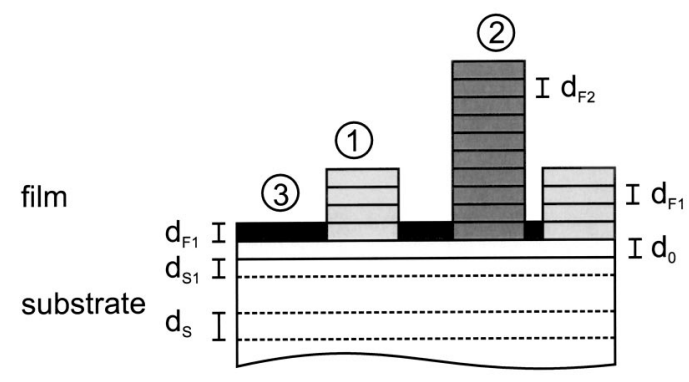

FIG. 5. Schematic of the model used for the calculation of the specular rod intensity. The film consists of three different regions: (1) Islands with a height corresponding to the average film thickness and the lattice constant $d_{F 1}$, (2) high islands with the lattice constant $d_{F 2}$, and (3) depleted areas with the lattice constant $d_{F 1}$. The distance between the first monolayer and the substrate, $d_{0}$, the lattice constant of the substrate, $d_{S}$, and the lattice constant of the topmost substrate layer, $d_{S 1}$, are indicated.
(MLS). After annealing up to $T_{a}=343 \mathrm{~K}$ [Fig. 3(b)], neighboring islands become clearly distinguishable. They are molecularly flat and develop characteristic facets as indicated in Fig. 3(b). The island height, $h$, approximately equals the average film thickness, $d$. For $T_{a} \geqslant 373 \mathrm{~K}$ [Figs. 3(c) and 3(d)], three morphological regions are discernible: (1) islands with $h \approx d$ and a lateral size of 50-200 nm, (2) islands with $h$ $\approx 3 d$ and a lateral size of $200-300 \mathrm{~nm}$, and (3) depleted areas [see Fig. 3(c)]. At $T_{a}=373 \mathrm{~K}$, many of the islands show facets, while the islands observed at $T_{a}=433 \mathrm{~K}$ appear to be isotropic.

\section{X-ray studies}

By way of example, we discuss a thin film with the growth parameters $T_{g}=235 \mathrm{~K}, \quad F=0.8 \AA / \mathrm{min}$, and $d$ $=50 \AA$. The sample has been annealed in three steps up to $308 \mathrm{~K}$, then in steps of $5 \mathrm{~K}$ up to desorption of the film. At each $T_{a}$, the sample has been annealed for 15-45 min. The specular rod measurement have been performed at $T_{a}$, after ensuring that no changes of the Bragg peak were visible during at least $10 \mathrm{~min}$.

Figure 4 shows examples of the specular rod measurements performed at different $T_{a}$. The measurements show several characteristic features: The asymmetric shape of the rod, the shape of the Bragg peak, and the Laue oscillations around the Bragg peak. For $T_{a}<358 \mathrm{~K}$, the number of Laue oscillations on either side of the Bragg reflection increases with temperature from one to three, indicating a decreasing roughness of the film. For $T_{a} \geqslant 358 \mathrm{~K}$, the number and periodicity of the Laue oscillations are independent of $T_{a}$. The Bragg peak observed for $T_{a} \leqslant 358 \mathrm{~K}$ is Gaussian in shape. It develops a second, narrower component for $T_{a}>358 \mathrm{~K}$. The peak width of the narrow component decreases with increasing $T_{a}$.

\section{Modeling of the x-ray data}

Based on the AFM observations, a model for the x-ray data has been developed. It is assumed that the sample consists of three types of mosaic blocks (Fig. 5): (1) Substrate mosaic blocks covered by a thin film with $n_{1}$ monolayers and the roughness $\sigma_{1}$, (2) substrate mosaic blocks covered by a thick smooth film with $n_{2}$ monolayers and a Gaussian height distribution of different mosaic blocks with the standard deviation $\sigma_{2}$, and (3) substrate mosaic blocks with residual coverages, $\theta_{n}$, of the individual layers. The type (1) mosaic blocks explain the Laue oscillations and the broad component of the Bragg peak, the type (2) mosaic blocks correspond to the sharp component, and the type (3) mosaic blocks are related to the shape of the underlying crystal truncation rod.

The lattice parameters and the different types of mosaic blocks are shown schematically in Fig. 5. The thin film covers the fraction $A_{1}$ of the total substrate surface, the thick film the fraction $A_{2}$, and the residual film the fraction $A_{3}$. $100 \%$ of the sample surface corresponds to the sum of $A_{1}$, $A_{2}$, and $A_{3}$. The scattering functions $S_{1}\left(q_{z}\right), S_{2}\left(q_{z}, n\right)$, and 
TABLE I. Parameters for the modeling of measurements of the specular rod at selected steps of $T_{a}$.

\begin{tabular}{lccccc}
\hline \hline Parameter & \multicolumn{5}{c}{} \\
\hline$T_{a}(\mathrm{~K})$ & 298 & 358 & 393 & 413 & 468 \\
$A_{1}(\%)$ & 98 & 60 & 33.9 & 37.1 & 32.6 \\
$A_{2}(\%)$ & 0 & 0 & 5.1 & 2.9 & 5.4 \\
$A_{3}(\%)$ & 2 & 40 & 61 & 60 & 62 \\
$d_{0}(\AA)$ & 2.85 & 2.95 & 2.85 & 2.80 & 2.85 \\
$d_{F 1}(\AA)$ & 3.252 & 3.258 & 3.273 & 3.281 & 3.302 \\
$d_{F 2}(\AA)$ & & & 3.267 & 3.270 & 3.289 \\
$n_{1}(\mathrm{ML})$ & 16 & 17.5 & 19.7 & 19.5 & 20 \\
$\sigma_{1}(\mathrm{ML})$ & 2.7 & 2.0 & 1.5 & 1.5 & 1.2 \\
$n_{2}(\mathrm{ML})$ & 0 & 0 & 26 & 45 & 40 \\
$\sigma_{2}(\mathrm{ML})$ & 0 & 0 & 26 & 45 & 40 \\
$\theta_{1}$ & 0 & 1 & 1 & 1 & 1 \\
$\theta_{2}$ & 0 & 0.6 & 0.4 & 0.4 & 0.4 \\
$\theta_{3}$ & 0 & 0.5 & 0.4 & 0.3 & 0.4 \\
$\theta_{4}$ & 0 & 0.1 & 0.15 & 0.2 & 0.15 \\
\hline \hline
\end{tabular}

$S_{3}\left(q_{z}\right)$ of the different types of mosaic blocks are calculated from the lattice sums of the substrate and the film, according to the kinematic scattering theory,

$$
\begin{aligned}
S\left(q_{z}\right)= & {\left[f_{S}\left(q_{z}\right)\left(\frac{1}{1-e^{-i q_{z} d_{S}}}+e^{i q_{z} d_{S 1}}\right) e^{-i q_{z}\left(d_{0}+d_{S 1}\right)}\right.} \\
& \left.+f_{F}\left(q_{z}\right) \sum_{k=1}^{\infty} \theta_{k} e^{i(k-1) q_{z} d_{F}}\right] e^{-\Delta q_{z}^{2} \sigma_{S}^{2} / 2},
\end{aligned}
$$

where $f_{S}\left(q_{z}\right)$ and $f_{F}\left(q_{z}\right)$ are the structure factors of the substrate and the film, $\sigma_{S}$ is the surface roughness of the substrate, $d_{S}$ is the lattice parameter of the substrate, $d_{S 1}$ is the lattice parameter of the topmost substrate layer, $d_{F}$ is the lattice parameter of the film, and $d_{0}$ is the distance between the topmost layer of the substrate and the first layer of the film. $\Delta q_{z}$ is the deviation of $q_{z}$ from the nearest substrate Bragg peak. The film morphology enters via $\theta_{k}$, which is the coverage of the layer $k$ within the film described by

$$
\theta_{k}=0.5\left[1-\operatorname{erf}\left(\frac{k-n_{1}}{\sigma_{1}}\right)\right]
$$

for the type (1) mosaic blocks,

$$
\theta_{k}= \begin{cases}1 & \text { for } k \leqslant n \\ 0 & \text { for } k>n,\end{cases}
$$

for the type (2) mosaic blocks, and

$$
\theta_{k}= \begin{cases}\geqslant 0 & \text { for } k \leqslant 4 \\ 0 & \text { for } k>4\end{cases}
$$

for the type (3) mosaic blocks. The total diffracted intensity is

$$
\begin{aligned}
I\left(q_{z}\right)= & A_{2} \frac{\sum_{n}\left(e^{-\left(n-n_{2}\right)^{2} / 2 \sigma_{2}^{2}}\left|S_{2}\left(q_{z}, n\right)\right|^{2}\right)}{\sum_{n}\left(e^{-\left(n-n_{2}\right)^{2} / 2 \sigma_{2}^{2}}\right)} \\
& +A_{1}\left|S_{1}\left(q_{z}\right)\right|^{2}+A_{3}\left|S_{3}\left(q_{z}\right)\right|^{2} .
\end{aligned}
$$

The Ag lattice parameters $d_{S}=2.36 \AA, d_{S 1}=2.34 \AA$, and the substrate roughness $\sigma_{S}=0.9 \AA$ have been determined by modeling the specular rod of the clean substrate. The sub- strate roughness refers to the lateral size of a mosaic block of typically $500 \AA$. The observed relaxation of the first ML corresponds to the results of studies using low-energy electron diffraction ${ }^{19}$ and medium-energy ion scattering. ${ }^{20}$ Within the experimental uncertainty, no variation of these parameters has been observed after depositing a thin PTCDA film. The local film thicknesses $\left(n_{2}, n_{1}\right)$, the lattice parameters $\left(d_{0}, d_{F 2}\right.$, and $\left.d_{F 1}\right)$, the coverages, $\theta_{k}$, of the type (3) mosaic blocks, the roughnesses $\left(\sigma_{1}\right.$ and $\left.\sigma_{2}\right)$, and the fractional areas $\left(A_{1}, A_{2}\right.$, and $\left.A_{3}\right)$ covered by different types of mosaic blocks have been varied. The lattice spacing, $d_{0}$, between the substrate and the first ML of the film is assumed to be the same for all types of mosaic blocks. The lattice parameters of the thin film, the residual coverage, $d_{F 1}$, and the lattice spacing of the thick film, $d_{F 2}$, are allowed to be different.

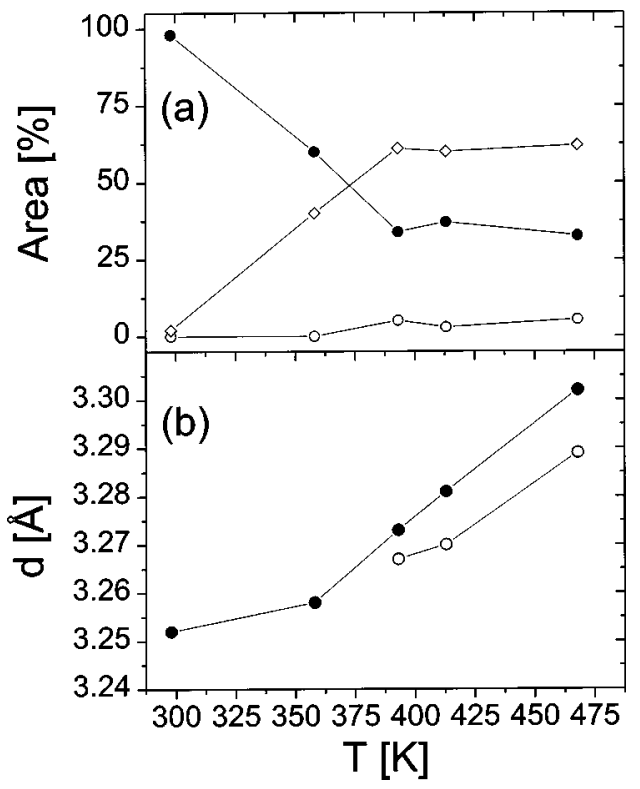

FIG. 6. (a) Area covered by the high mosaic blocks (open circles), by the thin-film mosaic blocks (filled circles), and by the residual coverage mosaic blocks (open diamonds) as a function of the annealing temperature. (b) Lattice constants as a function of the annealing temperature. Filled symbols: thin-film islands. Open symbols: high islands. 
The results of the modeling of the experimental data at selected $T_{a}$ are shown as solid lines in Fig. 4. Table I summarizes the corresponding parameters. For the measurements at $T_{a} \leqslant 358 \mathrm{~K}$, only two types of mosaic blocks are required for the modeling: The thin film and the residual coverage mosaic blocks. For $T_{a}>358 \mathrm{~K}$, all three types of mosaic blocks (thin film, thick film, and depleted areas) have been taken into account.

With increasing $T_{a}$, the ratio of the thin-film mosaic blocks decreases from nearly $100 \%$ to approximately $30 \%$, their thickness increases slightly from 16 to $20 \mathrm{ML}$, and their roughness decreases from approximately 2.7 to $1.2 \mathrm{ML}$. The thick-film mosaic blocks develop for $T_{a}>358 \mathrm{~K}$. They cover approximately $5 \%$ of the sample, their average height increases with $T_{a}$ up to three times the average thickness $d$, and they have a broad height distribution (due to the broad distribution, the values for $n_{2}$ and $\sigma_{2}$ should be treated as an estimate). The residual area is covered by approximately 2 ML PTCDA. The total amount of the coherently scattering material decreases $T_{a} \leqslant 358 \mathrm{~K}$ and stabilizes at approximately $65 \%$ of the initial value. We always find $d_{0}$ $\approx 2.85 \AA$, independent of $T_{a}$, which is less than both $d_{F 1}$ and $d_{F 2} . d_{0}$ is comparable to $0.5\left(d_{S}+d_{F}\right) \approx 2.80 \AA$. The lattice constants $d_{F 1}$ and $d_{F 2}$, with $d_{F 1}>d_{F 2}$, increase with $T_{a}$, as shown in Fig. 6 .

\section{Thermal expansion of bulk $\alpha$ 3,4,9,10-perylenetetracarboxylic dianhydride}

Figure 7 shows two powder spectra taken at 468 and 293 $\mathrm{K}$, respectively. Each spectrum averages over $10 \mathrm{~K}$ due to the integration over an area of the film used for detecting the $\mathrm{x}$-ray spectra. From the measurement, we have derived the thermal expansion coefficients $\alpha=5.47 \pm 0.39 \times 10^{-5} \mathrm{~K}^{-1}$ in the [011] direction, $\alpha=6.71 \pm 0.44 \times 10^{-5} \mathrm{~K}^{-1}$ in the [012] direction (both directions are nearly in the molecular plane), and $\alpha=1.240 \pm 0.022 \times 10^{-4} \mathrm{~K}^{-1}$ in the [102] direction (which is the stacking direction of the molecules). Though we cannot exclude possible systematic errors of at most 0.2 $\times 10^{-4} \mathrm{~K}^{-1}$ due to the misalignment of the film, the anisotropy of the thermal expansion parallel and perpendicular to the molecular plane is clearly visible.

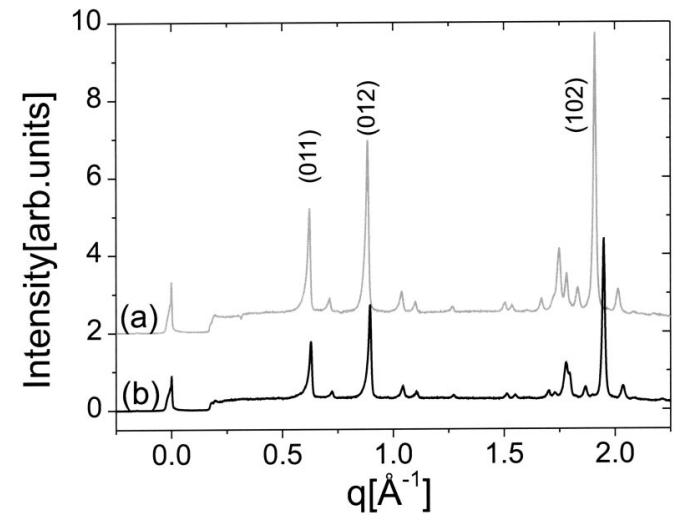

FIG. 7. Powder spectra of a bulk sample of $\alpha$ PTCDA; (a) measured at 468 $\mathrm{K}$ and (b) measured at $293 \mathrm{~K}$. The PTCDA (011), (012), and (102) peaks are indicated. (a)

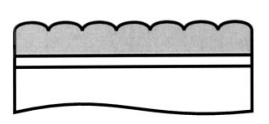

Film

Wetting layer

Substrate

(b)

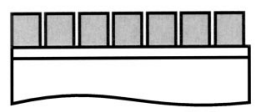

(c)

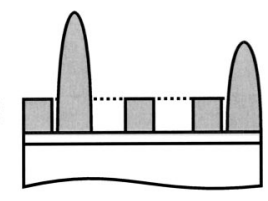

FIG. 8. Model for the partial dewetting. (a) The as-grown film with $T_{g}$ $\$ 350 \mathrm{~K}$ is smooth with a slightly corrugated surface. (b) During annealing up to $T_{a} \lesssim 350 \mathrm{~K}$, the film evolves into small islands with a smooth surface on top of a wetting layer. (c) During further annealing, a fraction of the film forms large islands, and the area covered only by the wetting layer increases, while the remaining film is stable.

\section{DISCUSSION}

As will be shown, the initially smooth low-temperature PTCDA films are metastable while the high-temperature island films are stable. In the following, both cases are discussed in detail.

The AFM data show that the relatively smooth PTCDA films grown at $T_{g} \lesssim 350 \mathrm{~K}$ are thermally unstable. The morphology changes from a film with a comparatively homogeneous height distribution to an inhomogeneous island distribution. A comparison with the x-ray experiments shows that the three types of mosaic blocks necessary for fitting the specular rod data correspond to the two different island sizes observed by AFM, and to the depleted regions inbetween the islands. The complementary information derived from the AFM and the $\mathrm{x}$-ray experiments leads to the following description of the partial dewetting of PTCDA/Ag(111), schematically shown in Fig. 8. The epitaxial PTCDA film smoothens during annealing up to approximately $350 \mathrm{~K}$, and the islands develop facets. During further annealing, the film dewets partially. The substrate is covered by a wetting layer of approximately 1-3 ML, islands with a height $h$ similar to the initial film thickness, $d$, and islands with $h \approx 3 d$. The two types of islands have different lattice constants and stay crystalline up to the desorption. The thermal expansion of both island types is $\alpha_{\perp}=1.0 \pm 0.2 \times 10^{-4} \mathrm{~K}^{-1}$ which is similar to the thermal expansion of the high-temperature films with $\alpha_{\perp}=1.05 \pm 0.06 \times 10^{-4} \mathrm{~K}^{-1}$. For $T_{a} \gtrsim 400 \mathrm{~K}$, the island size distribution appears to be stable on the time scale studied.

The thermal instability of the smooth film can be explained by several energetic contributions: The decrease of the surface energy by developing energetically favored facets, the decrease of the total energy of an island by increasing its volume and minimizing its surface (similar to observations made for PTCDA/glass), ${ }^{10}$ and the decrease of energy by increasing the ratio of the energetically favored polymorph. The first two aspects show directly in the morphology change, while the third aspect is supported by the fact that the high islands have a lower out-of-plane lattice constant than the low islands. The difference between the lattice constants corresponds to the difference between the $\alpha$ and the $\beta$ phase, and the smaller lattice constant of the high islands corresponds to the $\alpha$ polymorph energetically favored in the bulk. 
In contrast to the thermal instability of films grown at $T_{g} \lesssim 350 \mathrm{~K}$, the films grown at $T_{g} \geq 350 \mathrm{~K}$ (showing an island morphology) seem to be stable upon annealing for several hours although the island distribution might change during a longer-time annealing. The island morphology developed during the annealing of a smooth film seems to be related to the as-grown island morphology since the facets of both structures are similar, as well as the thickness of the wetting layer between the islands. Furthermore, the transition temperature from a smooth film to a partially dewetted surface is comparable to the transition temperature deduced from the transition of the as-grown smooth films to crystallites on a wetting layer. This suggests that both processes are determined by the same energy barrier, $E$. Based on this, we propose the following model. The initially smooth, metastable film is formed as a consequence of growth conditions far away from thermal equilibrium. For $T_{a} \lesssim 350 \mathrm{~K}$, the surface mobility is limited by $E$, and the only possible route for the energy minimization is the generation of facets similar to the as-grown crystallites. For $T_{a} \gtrsim 350 \mathrm{~K}$, the barrier can be overcome and high islands can grow.

While some of the PTCDA islands grow with increasing $T_{a}$, others disappear. We assume that the shrinking is controlled by surface diffusion. Most likely, the molecules from the top (102) surface diffuse to other islands since, at this surface, the molecules of the topmost plane do not overlap. It has been experimentally observed that the morphology is inhomogenous at high $T_{a}$. This indicates that not all of the low islands shrink with the same probability. The shrinking could be influenced by local effects including the substrate morphology, the influence of the island shape, the epitaxial orientation, and the crystalline structure of neighboring islands.

Morphology changes similar to the ones observed for PTCDA have been reported for several inorganic thin films. For instance, similar to the system PTCDA/Ag(111), Mn/ $\mathrm{Fe}(001)$ prefers the Stransky-Krastanov growth mode for substrate temperatures higher than the transition region. For these films, it has also been observed that smooth metastable films grown at temperatures below the transition region partially dewet upon annealing. ${ }^{21}$

For the high-temperature films, an anisotropic thermal expansion, parallel and perpendicular to the molecular plane, has been observed. The linear thermal expansion coefficient of $\mathrm{Ag}$ in the temperature range studied here is approximately $2 \times 10^{-5} \mathrm{~K}^{-1}$, which is smaller than the in-plane thermal expansion coefficient of bulk PTCDA. Moreover, the observed in plane unit cell is rectangular, as expected from a relaxed bulk structure, while epitaxial strain is expected to induce deviations from the rectangular unit mesh. This indicates that the observed thermal expansion is not dominated by lattice clamping. In addition, the thermal expansion coefficients measured on the thin films are similar to the bulk data. The anisotropic thermal expansion is typical for planar molecules and has also been observed, e.g., for several TCNQ salts ${ }^{5}$ and oligothiophenes. ${ }^{22}$

\section{SUMMARY AND CONCLUSIONS}

It has been observed that comparatively smooth PTCDA films deposited on $\operatorname{Ag}(111)$, at growth temperatures lower than approximately $350 \mathrm{~K}$, are metastable. The temperatureinduced transition to a partially dewetted morphology seems to be related to the morphology transition of as-grown films as a function of the growth temperature.

Metastable metal films of a similar type are known to grow under growth conditions far from thermal equilibrium. ${ }^{23}$ This observation indicates that the basic principles of organic and metal thin films exhibit certain similarities. The metastability of (organic) thin films is of great technical relevance since it limits the operational conditions for thin-film devices before degradation, but may be also used to tailor lateral structures, such as, e.g., quantum dots. ${ }^{6}$

\section{ACKNOWLEDGMENTS}

The authors are grateful to N. Karl for providing the purified material, and to P. Dietzel for help with the thermal expansion measurements of the bulk samples. Partial support from the DFG (Schwerpunktprogramm "Organische Feldeffekt-Transistoren") is gratefully acknowledged. The authors thank the HASYLAB staff for excellent support.

${ }^{1}$ S. R. Forrest, Chem. Rev. (Washington, D.C.) 97, 1793 (1997).

${ }^{2}$ N. Karl and C. Günther, Cryst. Res. Technol. 34, 243 (1999).

${ }^{3}$ A. C. Dürr, F. Schreiber, M. Kelsch, H. D. Carstanjen, and H. Dosch, Adv. Mater. (Weinheim, Ger.) 14, 961 (2002).

${ }^{4}$ S. Dietrich, Phase Transitions and Critical Phenomena (Academic, London, 1988), Vol. 12.

${ }^{5}$ S. van Smaalen, J. de Boer, C. Haas, and J. Kommandeur, Phys. Rev. B 31, 3496 (1985).

${ }^{6}$ V. A. Shchukin and D. Bimberg, Rev. Mod. Phys. 71, 1125 (1999).

${ }^{7}$ K. Glöckler, C. Seidel, A. Soukopp, M. Sokolowski, E. Umbach, M. Böhringer, R. Berndt, and W.-D. Schneider, Surf. Sci. 405, 1 (1998).

${ }^{8}$ F. S. Tautz, E. Eremtchenko, J. A. Schaefer, M. Sokolowski, V. Shklover, and E. Umbach, Phys. Rev. B 65, 125405 (2002).

${ }^{9}$ G. Salvan, D. A. Tenne, A. Das, T. U. Kampen, and D. R. T. Zahn, Organic Electronics 1, 49 (2000).

${ }^{10}$ S. Heutz, A. J. Fergusio, G. Rumbles, and T. S. Jones, Organic Electronics 3, 119 (2002).

${ }^{11}$ B. Krause, A. C. Dürr, K. A. Ritley, F. Schreiber, H. Dosch, and D. Smilgies, Appl. Surf. Sci. 175, 332 (2001).

${ }^{12}$ B. Krause, A. C. Dürr, K. Ritley, F. Schreiber, H. Dosch, and D. Smilgies, Phys. Rev. B 66, 235404 (2002).

${ }^{13}$ A. J. Lovinger, S. R. Forrest, M. L. Kaplan, P. H. Schmidt, and T. Venkatesan, J. Appl. Phys. 55, 476 (1984).

${ }^{14}$ M. L. Kaplan, C. S. Day, A. J. Lovinger, P. H. Schmidt, and S. R. Forrest (private communication).

${ }^{15}$ M. Möbus, N. Karl, and T. Kobayashi, J. Cryst. Growth 116, 495 (1992).

${ }^{16}$ T. Ogawa, K. Kuwamoto, S. Isoda, T. Kobayashi, and N. Karl, Acta Crystallogr., Sect. B: Struct. Sci. 55, 123 (1999).

${ }^{17}$ K. Ritley, B. Krause, F. Schreiber, and H. Dosch, Rev. Sci. Instrum. 72, 1453 (2001)

${ }^{18}$ Y. S. Touloukian, R. K. Kirby, R. E. Taylor, and P. D. Desai, Thermophysical Properties of Matter (IFI/Plenum, New York, 1975), Vols. 12 and 13.

${ }^{19}$ E. A. Soares, G. S. Leatherman, R. D. Diehl, and M. A. van Hove, Surf. Sci. 468, 129 (2000).

${ }^{20}$ P. Statiris, H. C. Lu, and T. Gustafsson, Phys. Rev. Lett. 72, 3574 (1994).

${ }^{21}$ R. Pfandzelter, T. Igel, and H. Winter, Surf. Sci. 389, 317 (1997).

${ }^{22}$ F. J. Ramírez, M. A. G. Aranda, V. Hernández, J. Casado, S. Hotta, and J. T. L. Navarrete, J. Chem. Phys. 109, 1920 (1998).

${ }^{23}$ E. Bauer and J. H. van der Merwe, Phys. Rev. B 33, 3657 (1986). 